\title{
Restructuring of the Ge(100) surface by Na chains
}

\author{
W. Ernst, ${ }^{1}$ K.-L. Jonas, ${ }^{2}$ V. von Oeynhausen, ${ }^{2}$ C. Tegenkamp, ${ }^{1}$ and H. Pfnür ${ }^{1, *}$ \\ ${ }^{1}$ Institut für Festkörperphysik, Universität Hannover, Appelstraße 2, D-30167 Hannover, Germany \\ ${ }^{2}$ Fachbereich Physik, Universität Rostock, Universitätsplatz 3, D-18055 Rostock, Germany
}

(Received 8 April 2003; published 6 November 2003)

\begin{abstract}
The Na-induced surface structures on $\mathrm{Ge}(100)$ at concentrations below 0.1 monolayer (ML) have been investigated with low-energy electron diffraction and with scanning tunneling microscopy (STM). Annealing and partial desorption of higher coverages at temperatures between 650 and $680 \mathrm{~K}$ lead to the formation of characteristic chain structures, alternating between uncovered Ge dimer or double dimer rows and Na chains. The $\mathrm{Na}$ atoms have a preferential distance of three Ge lattice constants along the chains, but there is little correlation between different chains. Locally, only commensurate distances between Na chains of 4, 6 and $8 \mathrm{Ge}$ lattice constants have been detected, with average distances being either commensurate or incommensurate, depending on the Na concentration. STM suggests complete removal of Ge-dimer rows below adsorbed Na chains. These alkali induced chainlike and well ordered self-organized modulations can be used as templates to impose these structures onto adsorbed ultra-thin insulating films.
\end{abstract}

DOI: 10.1103/PhysRevB.68.205303 PACS number(s): 71.20.Dg, 61.72.Tt, 68.35.-p, 61.14.Hg

\section{INTRODUCTION}

Alkali metals adsorbed on semiconductor surfaces have attracted wide interest in the past in context with both applied and basic physical phenomena. Just to mention two examples of applications, semiconductors covered with alkali metals have been used as infrared detectors, ${ }^{1}$ and also to speed up the oxidation of silicon and germanium. ${ }^{2-5}$

Adsorption induced reduction of symmetry, on the other hand, is a general phenomenon seen as formation of various adsorbate induced superstructures. The peculiarity of metal adsorption, e.g., on $\mathrm{Si}(111)^{6-8}$ and for adsorption of sodium on $\operatorname{Si}(100)^{9,10}$ is the formation of chain structures, which have in part remarkably large periodicities between the chains. The coupling mechanism has been clarified only in some cases. For In/Si(111), ${ }^{11,12}$ e.g., electron-phonon coupling together with a large adsorbate induced anisotropy and a nesting of the Fermi surface lead to formation of an adsorbate induced charge density wave. It has been identified as the basic mechanism for structure formation and the related phase transitions as well as for the metal-insulator phase transition coupled with the structural changes. ${ }^{13}$ These transitions can also be modified by coadsorbed alkali atoms. ${ }^{14}$ The structures investigated here, however, do not seem to be related to these phenomena for reasons discussed below.

A motivation aimed more towards structural manipulation is the use of strongly modulated self-organized surfaces for the controlled manipulation or modification of film morphologies grown on these surfaces. As a recent example, we reported the influence of a defined Na precoverage on growth and morphology of thin insulating films of $\mathrm{NaCl}$ on $\mathrm{Ge}(100) .{ }^{15}$ We showed that regularly arranged sodium atoms located at the interface between the film and the substrate lead to the formation of a one dimensional, periodic, and vertical modulation of the insulating layer. For clarification of the underlying mechanisms the structural properties of such modulated surfaces are the first steps of investigation.

The system under investigation here has not been studied yet in great detail, although both theoretical ${ }^{16}$ and experi- mental investigations exist. ${ }^{17,18}$ With respect to the structure, there is similarity with the system $\mathrm{Na} / \mathrm{Si}(100) .^{9,10}$ In both systems a metastable $p(4 \times 1)$ superstructure is formed during dosing at room temperature, which is replaced by a $p(2 \times 1)$ phase upon further evaporation of sodium. No details are known for the low-coverage ordered phases of the $\mathrm{Na} / \mathrm{Ge}(100)$ system investigated here. Here we concentrate on the periodic, but anisotropic surface rearrangements and reconstructions with large unit cells induced by adsorbed $\mathrm{Na}$ in the low coverage regime below 0.1 ML. These structures are observed after coupled annealing and partial desorption of $\mathrm{Na}$, starting from higher coverages. Based on our lowenergy electron diffraction (LEED) and scanning tunnel microscope (STM) results, we derive a geometric model of the local adsorption geometry for the chain structures found, discuss the influence of these structures on step morphology and potential applications.

\section{EXPERIMENT}

LEED measurements were carried out in a UHV chamber, which is equipped with photoelectron spectroscopy [ ultraviolet photoemission spectroscopy (UPS) and x-ray photoemission spectroscopy (XPS)], electron-loss spectroscopy (EELS), LEED, and a mass spectrometer, at a base pressure below $10^{-8} \mathrm{~Pa}$. Ge(100) single crystals (Crystec, Berlin) were polished mechanically with diamond pastes followed by a final chemical treatment. The miscut angle was specified to be better than $0.2^{\circ}$. These crystals were mounted on a transferable sample holder, which allowed heating both by direct current and by radiation/electron bombardment using a filament located behind the sample. The temperature was controlled by a Ni/Ni-Cr thermocouple attached to the sample holder.

In UHV the sample was degassed at $600 \mathrm{~K}$ for at least 12 h. Five to ten cycles of Argon sputtering and annealing followed. The sample was bombarded at room temperature with ions at $900 \mathrm{eV}$ kinetic energy at a typical current density of $1.5 \mu \mathrm{A} / \mathrm{cm}^{2}$. Subsequently, the sample was annealed at 850 - 


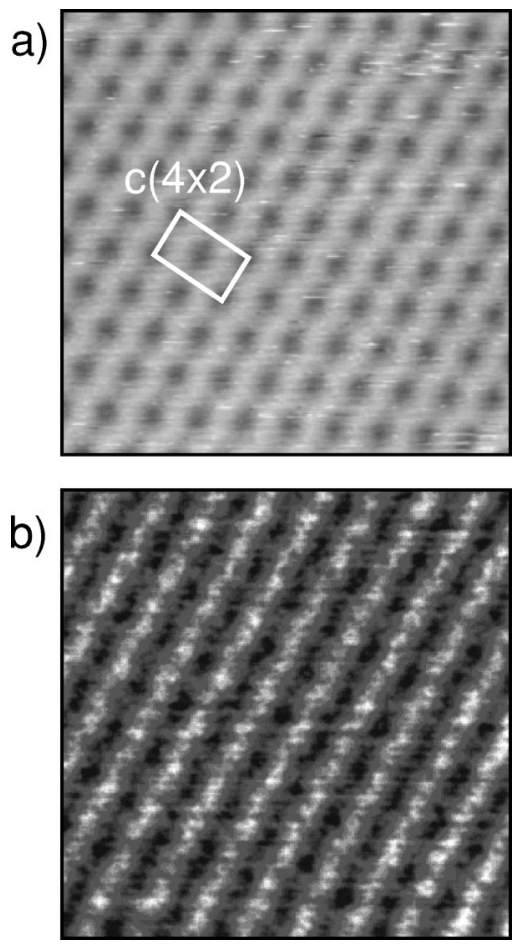

FIG. 1. Filled (top) and empty (bottom) state STM images of clean $\mathrm{Ge}(100)$. Tunneling conditions: $-1.6 \mathrm{~V}, 0.3 \mathrm{nA}$ (top), $+1.6 \mathrm{~V}$, $0.3 \mathrm{nA}$ (bottom).

$900 \mathrm{~K}$ by direct current heating for $30 \mathrm{~min}$. After cooling down to room temperature the samples showed a brilliant $p(2 \times 1)$ LEED pattern with sharp spots and a low background signal. At temperatures below $200 \mathrm{~K}$ sharp and intense spots of the $c(4 \times 2)$ structure were observed. Between these cycles the cleanness was controlled by XPS and Auger electron spectroscopy (XAES), and the procedure was repeated until no impurities (especially carbon) were detected.

Sodium was evaporated from a well degassed dispenser (SAES Getters). During adsorption of $\mathrm{Na}$ the sample was at room temperature for most experiments. For annealing, the sample was either heated by direct current or by radiation using a filament located directly behind the sample.

STM measurements were performed in a UHV system with a low-temperature STM supplied by Omicron. It consists of two chambers, one containing the STM (base pressure $10^{-9} \mathrm{~Pa}$ ) and a second for sample preparation and LEED measurements (base pressure $10^{-8} \mathrm{~Pa}$ ). The sample preparation was same as described above, except that the crystal was heated by electron bombardment in this case. Average terrace widths of $500 \AA$ are typical for these samples, as checked with STM. During all STM measurements the sample temperature was $80 \mathrm{~K}$, i.e., measurements of the clean $\mathrm{Ge}(100)$ sample revealed a completely $c(4$ $\times 2$ ) reconstructed surface.

\section{RESULTS AND DISCUSSION}

STM images of the pure Ge(100) surface in constant current mode are shown in Fig. 1 for reference and for comparison with those found for the $\mathrm{Na}$ covered surfaces below. At

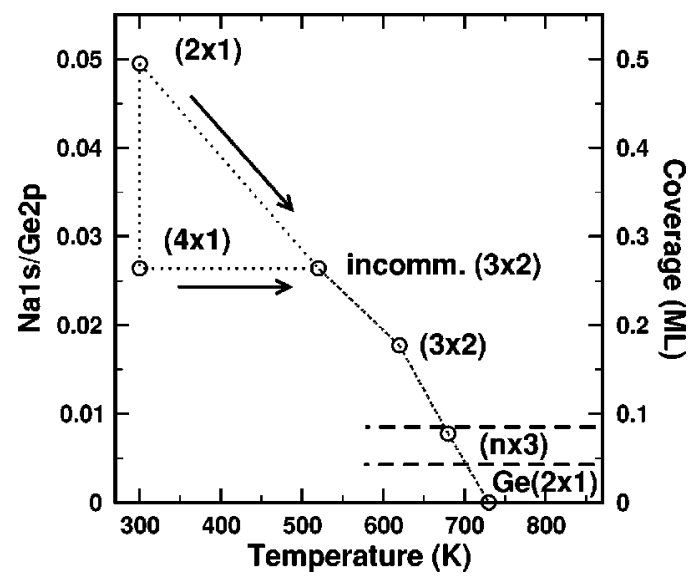

FIG. 2. Diagram of the surface structure as a function of coverage and annealing history.

the measuring temperature of $80 \mathrm{~K}$, a $c(4 \times 2)$ is formed out of the asymmetric dimers. This structure can clearly be resolved, when the tunneling current is emitted from the occupied states of the sample (top panel), whereas tunneling into the unoccupied Ge states only yields stripelike height variations. We will use these images later as a kind of finger print for uncovered and electronically unaltered parts of the $\mathrm{Ge}(100)$ surface.

$\mathrm{Na}$ adsorbed on this surface yields a wealth of structures. The Na-induced structures are briefly summarized in Fig. 2. More details are discussed in Ref. 19, which also discusses the absolute coverage calibration. During adsorption at room temperature, $(4 \times 1)$ and $(2 \times 1)$ structures appear at quarter and half monolayer (ML) coverage, respectively. At this concentration the first layer saturates. (The concentration of one monolayer corresponds to the same atom density as the first layer of the Ge substrate.) Thicker layers grow in StranskiKrastanov mode as three-dimensional islands. Starting from the monolayer, partial desorption yields new incommensurate structures close to quarter monolayer coverage, but no $(4 \times 1)$ structure reappears, an indication that this structure is metastable. Further thermal desorption results in the formation of the commensurate $(2 \times 3)$ structure, which contains only one $\mathrm{Na}$ atom per unit cell, as revealed by STM. This is the first commensurate LEED structure generated by thermal activation on this surface. This thermal activation is coupled with a rearrangement of substrate atoms in the first layer(s), as has already been discussed in Ref. 19. As we will show in this paper, where we concentrate on the annealed lowcoverage phases, further details can be learned about the basic Na-induced local structure by studying the phases at coverages below 0.1 ML.

When the Na layers are annealed above $630 \mathrm{~K}$, where the $(2 \times 3)$ structure has been found, the Na coverage is further reduced, but only in the temperature range between 660 and $680 \mathrm{~K}$, clear and well ordered new LEED patterns have been observed. Examples are shown in Fig. 3, where sharp and well ordered $(4 \times 1)$ and $(6 \times 1)$ structures, ordered as two domains rotated by $90^{\circ}$, can be seen. At the $1 / 3$ order positions, however, characteristic streaks appear in both LEED patterns of Fig. 3, indicating additional short-range order 

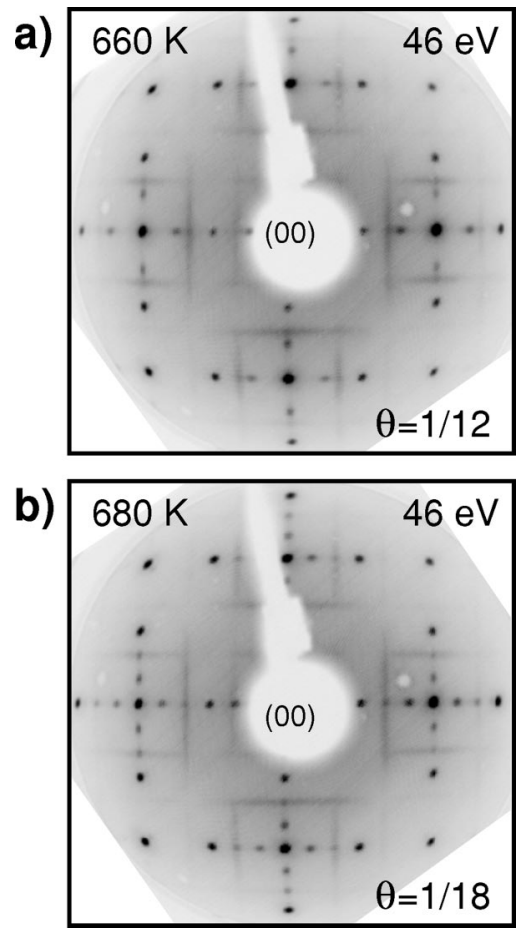

FIG. 3. LEED patterns, taken at room temperature, found after partial desorption for residual coverages 0.085 (top) and 0.055 (bottom). The most intense (darkest) spots are the first-order spots of the substrate.

with a characteristic distance of $3 a$ [ $a$ is the lattice constant of the $\mathrm{Ge}(100)$ substrate] in the direction perpendicular to the fourfold or sixfold periodicity, respectively. Neglecting any structure within these streaks, the streaks look like the superposition of the scattered intensity from chains of point scatterers, e.g., atoms (or an array of incoherently scattering chains) with a lateral distance of $3 a$ within the chain. If the chain atoms are located on lattice sites of the substrate, however, and are separated by periodic distances $n a$, corresponding to the dominant superstructure, the correlation between different chains for lattice sites (but not for distances $3 a$ ) remains, and scattering is coherent and in phase for integer order as well as fractional-order diffraction conditions of a $(n \times 1)$ structure. This leads to a concentration of diffracted intensity in the integer order beams and the fractional-order beams between them, as observed, and the disappearance of the streaks between integer order beams. More details of this structure will be clarified with STM described below. Taking the local order as most characteristic, these structures will be classified as $(n \times 3)$ structures with $n=4$ and $n=6$ for the structures shown in Fig. 3, although long-range order exists only in one direction.

Incommensurate $(n \times 3)$ structures with fractional $n$ between 4 and 8 have been obtained by appropriate partial desorption of residual layers, i.e., the average distance between structural units can be uniaxially adjusted by coverage. Taking the $(2 \times 3)$ structure with a single $\mathrm{Na}$ atom per unit cell as reference (i.e., Na coverage of $1 / 6)$, the $(4 \times 3)$ corresponds to a coverage of $1 / 12$, whereas the $(6 \times 3)$ structure is formed at a concentration of only 1/18 ML. In order to a)

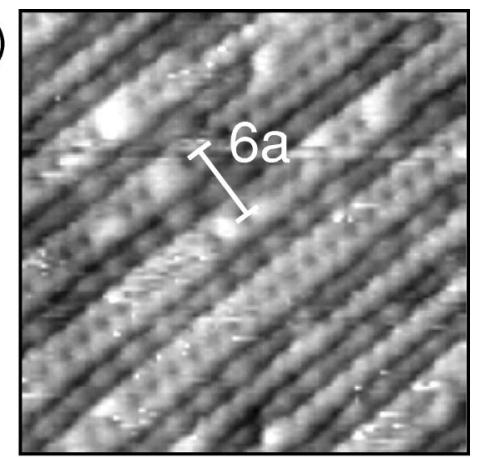

b)

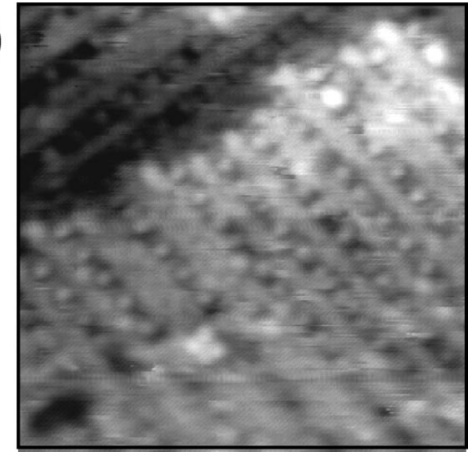

FIG. 4. Enlarged sections of the $(6 \times 3) \mathrm{Na} / \mathrm{Ge}(100)$ surface for both positive (bottom) and negative tunneling voltages. Tunneling conditions: $-1.3 \mathrm{~V}, 0.82 \mathrm{nA}$ (top) and $+1.2 \mathrm{~V}, 0.42 \mathrm{nA}$ (bottom).

get further information about details of the local structure, we performed STM measurements at $80 \mathrm{~K}$ on Na covered $\mathrm{Ge}(100)$ surfaces that have been first characterized by LEED. As an example, Fig. 4 shows STM images in constant current mode at an average $\mathrm{Na}$ concentration of $0.065 \mathrm{ML}$ for tunneling out of occupied Ge states (top) and into unoccupied states of the Ge surface (bottom).

As seen in Fig. 4(b), and in agreement with the results from LEED, the annealed layers of Na form strongly anisotropic structures with two domains rotated by $90^{\circ}$ on different $\mathrm{Ge}(100)$ terraces that are separated by steps of monatomic height. Atomic resolution has been partly achieved, especially when tunneling from the occupied Ge states [Fig. 4(a)]. When comparing this figure with Fig. 1, it is obvious that the broad braidlike structures resemble very closely to those observed for the $c(4 \times 2)$ structure of the clean surface, i.e., this structure locally reappears as chains of double-dimer rows at this Na coverage. In agreement with this assignment, we observe much less structured stripes when the unoccupied Ge states are involved in the tunneling process.

These double-dimer rows are separated by chains with only one protrusion and a typical separation of $3 a$ between them along the chains. They can be seen with approximately the same resolution for both positive and negative tunneling voltages. When comparing these structures with those obtained for the $(2 \times 3)$ structure, ${ }^{19}$ it is fully consistent to identify these protrusions with the positions of the $\mathrm{Na}$ atoms, and these chains with $\mathrm{Na}$ chains that have an average separation of $3 a$ along the chains. Na chains separated by a double-dimer row of Ge must have a lateral distance of $6 a$. Since the Na coverage chosen in Fig. 4 exceeded the optimal 


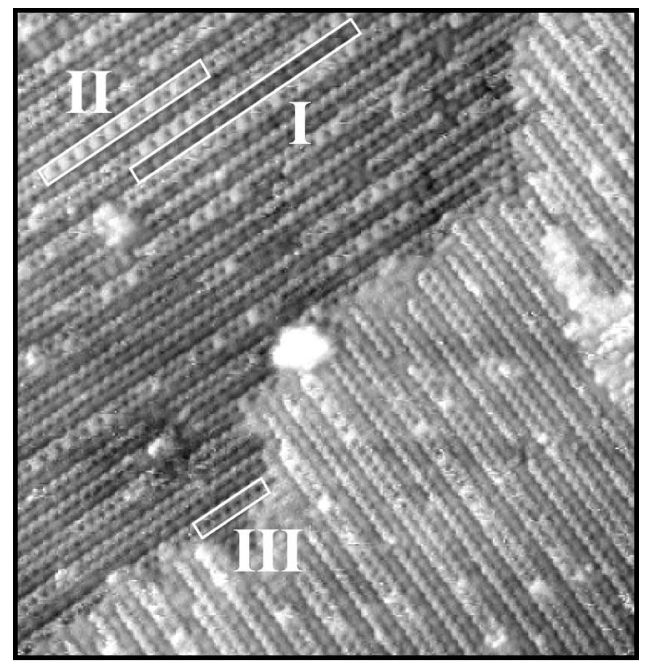

$\begin{array}{llllllll}0 & 24 & 48 & 72 & 96 & 120 & 144 & 168\end{array}$

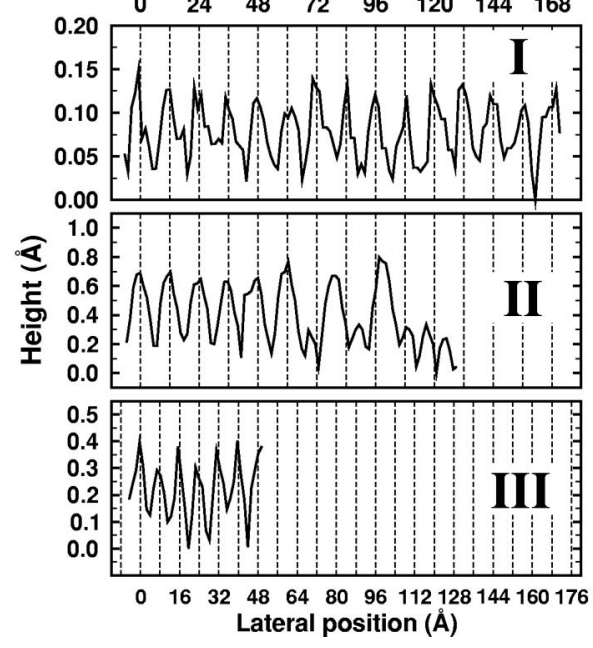

FIG. 5. Overview by STM of the Na-induced $(6 \times 3)$ structure (top) together with selected line scans (lower panel) along the sections marked I, II, and III.

coverage for the $(6 \times 3)$ structure, we also observe Na chains that are separated by a single-dimer row of Ge. These are separated by $4 a$. This justifies our nomenclature of the structures.

The lateral distances along the chains have been checked in more detail using Fig. 5. This analysis also shows that there are in fact two different Na-induced modulations on the surface. In addition to those, which we have already described as $\mathrm{Na}$ chain in between dimer or double-dimer rows of Ge, there is a minority species, mainly on top of the double dimer rows, seen as light protrusions in Fig. 5. The lateral distances of the various structures along the Ge-dimer rows have been measured by determining the height modulation function in this direction at various locations marked I, II, and III in Fig. 5, respectively. Along part I, which we have identified with a chain of single $\mathrm{Na}$ atoms, regular modulations with a period of approximately $12 \AA$ are seen. This corresponds to three Ge lattice constants, as expected. The same period, although with more stacking faults is found in Sec. II, which corresponds to the $\mathrm{Na}$ minority species on top of the double-dimer rows. In Sec. III we measured on a bare double-dimer row, and found the periodicity of $2 a$, as expected for a local $c(4 \times 2)$ structure in this direction.

Thus it is obvious that in annealed low coverage Na layers on $\mathrm{Ge}(100)$ the most stable basic unit are chains of single $\mathrm{Na}$ atoms parallel to the original Ge dimers at a distance of $3 a$. Although STM cannot prove in a strict sense the replacement of Ge-dimer rows by $\mathrm{Na}$ chains, the characteristic height modulations found in our images strongly suggest that $\mathrm{Na}$ has essentially replaced the dimer rows of $\mathrm{Ge}$, so that the $\mathrm{Na}$ atoms are on a height level close to the original Ge dimers. Normal to the chains, only a preference for chain distances of $n \times a$ with $n=4,6$ can be derived, i.e., a preference for integer units of dimers. The average distance, on the other hand, is adjusted by $\mathrm{Na}$ concentration. There are no indications for phase separations into regions with $6 a$ and $4 a$, which shows that the chain-chain interaction is purely repulsive. Na must be adsorbed on well defined lattice sitesotherwise we would not be able to determine any periodicity along the $\mathrm{Na}$ chains. From this information, the LEED patterns seen can be easily reconstructed. Assuming no correlations between the $\mathrm{Na}$ chains apart from their separation and their adsorption on lattice sites, the periodic units of $n / 2$ Ge-dimer rows plus a $\mathrm{Na}$ chain form a $(n \times 1)$ LEED pattern. The long streaks at $1 / 3$ order positions in the LEED patterns shown in Fig. 3 reflect the threefold periodicity along single $\mathrm{Na}$ chains, as seen in our STM images. The small short-range correlations between the $\mathrm{Na}$ positions on different chains, expressed by small intensity modulations along the streaks in LEED, is hard to be detected by STM. Thus there is complete agreement between LEED and STM. For perfect correlations between the $\mathrm{Na}$ chains, a $(6 \times 3)$ structure would be seen.

The minority species of Na chains (Sec. II of Fig. 5) located on the double-dimer rows of Ge exhibits under the tunneling conditions chosen in this figure the same periodicity along the chains, but a higher corrugation than majority species. This is an indication of different local electronic distributions for the two species that are adsorbed in different local environments of Ge atoms. Although the STM images suggest that the Ge dimer structure underneath there Na species are distorted only very locally, and may even still be there, the $\mathrm{Na}$ atoms in these chains, which look all equivalent in STM, must cause some relaxations, since they impose their own new periodicity onto the Ge rows.

Although the local adsorption sites cannot be directly determined from our STM results nor from qualitative LEED, we try to give a plausible structural model for the $n \times 3$ structures. To be specific, we restrict ourselves to the $(6 \times 3)$ unit cell in Fig. 6, and to a discussion of the majority species of $\mathrm{Na}$. Within the $\mathrm{Na}$ chains, we see no indications for remaining Ge dimers, irrespective of the tunneling conditions used. Therefore, we assume that whole rows of Ge dimers of the first layer are replaced by the $\mathrm{Na}$ chains during the annealing process. The essentially symmetric intensity distribution along the chains also suggests a symmetric adsorption site with respect to the remaining Ge environment. This could either be a pedestal site with four Ge neighbors in the next layer, or a bridge site with only two Ge neighbors in the next layer. The latter position, however, would still allow forma- 

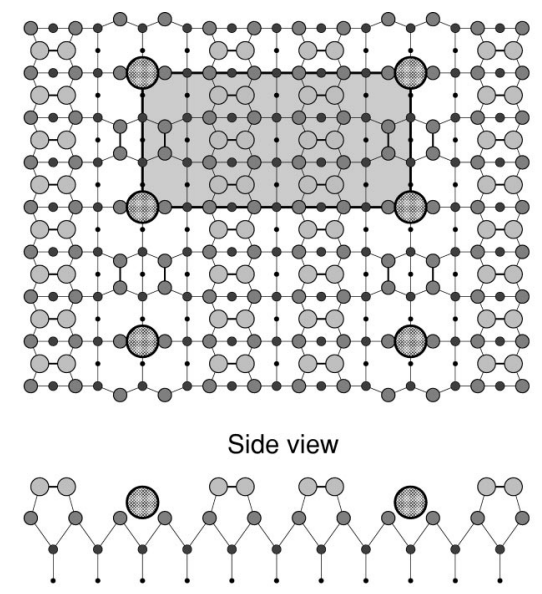

$\mathrm{Na} \bigcirc \operatorname{layer} 1(\mathrm{Ge}) \quad \circ$ layer $2 \cdot$ layer $3 \cdot$ layer 4

FIG. 6. Structure model for the $p(6 \times 3) / \mathrm{Na} / \mathrm{Ge}(100)$ reconstruction. Please note that in reality the $\mathrm{Na}$ chains are only poorly correlated.

tion of additional Ge dimers along the chains, and may thus compensate for the reduced $\mathrm{Na}$ coordination. While details can only be clarified by quantitative calculations, this structural model can be viewed as a natural extension of the $(2$ $\times 3$ ) structure, ${ }^{19}$ which corresponds to $n=2$ and has no Gedimer rows between the $\mathrm{Na}$ chains. It should be noted, as already discussed in context with the LEED results above and also seen in the STM images, that in contrast to the simplified local drawing of Fig. 6, very little correlation exists between different $\mathrm{Na}$ chains in the direction along the $\mathrm{Na}$ chains, i.e., the figure is only meant to illustrate the local configuration. With this restriction, the model is fully compatible both with the LEED and with the STM results. The periodicity of $n=6$, etc. is produced by the periodic arrangement of double (single) Ge-dimer rows coupled with single $\mathrm{Na}$ chains, whereas the streaks in LEED at the 1/3 order positions are due to the periodicity of $3 a$ within the $\mathrm{Na}$ chains, as already mentioned.

The high annealing temperatures, leading to partial desorption of the layers, are obviously necessary to obtain the structures reported here. The most likely reason for the high activation energy associated with the formation of these structures is the necessary transport of Ge atoms of the first $\mathrm{Ge}$ layer during the formation of the Na chain structure. The large energy of activation also makes the pronounced metastability of other structures plausible, e.g., of the $(4 \times 1)$ structure formed at room temperature. This is a likely reason why the structure proposed here according to our STM results (see Fig. 6) disagrees with local structure of Na determined with surface x-ray diffraction. ${ }^{18}$ In this paper, it is shown that after room temperature adsorption of $0.3-0.6$ ML, two different adsorption sites in the valleys between the Ge-dimer rows are occupied by Na, i.e., the Ge-dimer structure stays intact in this case. While these results clearly contradict the calculations carried out in Ref. 16, the site found by us for the minority species on the double-dimer rows, may in fact be close to the adsorption site determined with $\mathrm{x}$-ray diffraction. If this interpretation is correct, it would

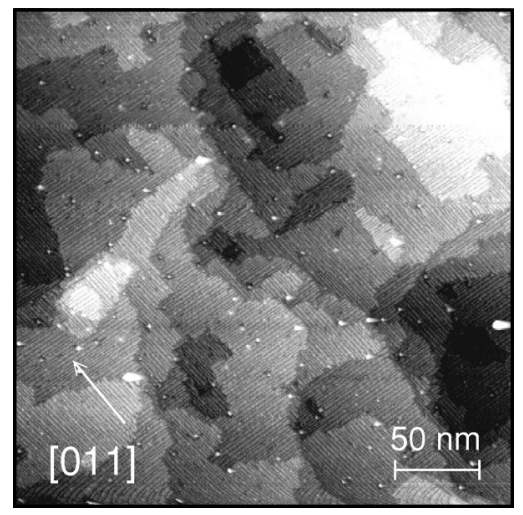

FIG. 7. Overview STM picture of the $\mathrm{Ge}(100)$ surface covered with a $\mathrm{Na}(6 \times 3)$ structure. Note that, in contrast to the $(2 \times 3)$ covered surface, no anisotropy of the step structure is seen.

provide evidence for the existence of a metastable species, which exists as minority species at high temperatures, presumably with the local geometry determined in Ref. 18, together with the more stable species described above in detail. While entropic effects may play a role that are not taken into account in calculations at $T=0,{ }^{16}$ our results also show that effective lateral interactions over more than three lattice constants are important for the structures observed, which have not been taken into account in the calculations with fairly small Ge clusters. ${ }^{16}$

Our model of the annealed Na-induced surface reconstruction with removal of $\mathrm{Ge}$ dimer rows below the $\mathrm{Na}$ chain is further supported by the corrugation function determined with LEED for epitaxial $\mathrm{NaCl}$ layers adsorbed on these $\mathrm{Na}$ modified Ge surfaces. ${ }^{15}$ As shown in Ref. 15, the onedimensional chain structure induced by $\mathrm{Na}$ at a coverage of 1/18 ML can be imposed onto the $\mathrm{NaCl}$ layer, resulting in $(6 \times 1)$ modulation with mainly a height corrugation and an amplitude of about $0.3 \AA$ for a $10 \mathrm{ML}$ thick NaCl film. Two dips can be identified in this height corrugation function, a small one associated with the uncovered double-dimer rows of $\mathrm{Ge}$ and a large one associated with the Na chains, which form a stronger bond with the $\mathrm{Cl}$ ions of the $\mathrm{NaCl}$ layer than the Ge atoms. The small amplitude of the corrugation function means that $\mathrm{Na}$ has to be bound to the Ge surface at a very similar height level as the remaining topmost $\mathrm{Ge}$ atoms of the dimer rows, that is, both the amplitude of the corrugation and the double-dip structure of the corrugation function would be hardly understandable if the $\mathrm{Na}$ chains are adsorbed on or between intact Ge dimer rows. In other words, these findings also favor the model of removal of complete Ge-dimer rows below the Na chains.

In contrast to the $(2 \times 3)$ structure investigated recently, ${ }^{19}$ the step structure on the Ge(100) surface is not strongly influenced by the residual $\mathrm{Na}$ concentration of less than 0.1 ML, see Fig. 7. Only the asymmetry between rough $A$-type and smooth $B$-type steps has disappeared. In fact, the step structure is almost isotropic, only showing preferential orientations given by the symmetry of the $\mathrm{Ge}(100)$ surface. This demonstrates that contributions from the dimer structure of the $\mathrm{Ge}(100)$ substrate again dominate the step formation energies and also the step-step interaction at these low Na con- 
centrations. In other words, the Na chains in this case do not make the step-step interactions already present on the clean $\mathrm{Ge}(100)$ surface more anisotropic.

\section{SUMMARY AND CONCLUSIONS}

We have investigated with LEED and STM the surface modifications of the Ge(100) surface induced by low coverages of $\mathrm{Na}$ after annealing and partial desorption of $\mathrm{Na}$ at temperatures between 650 and $680 \mathrm{~K}$. Our experiments show that this treatment of thermal activation triggers complete surface reconstruction into chainlike structures with alternating rows of uncovered $\mathrm{Ge}$ dimers and $\mathrm{Na}$ chains. The $\mathrm{Na}$ adsorbed in these chains has a preferential distance of $3 a$, but little correlations between different chains have been found, resulting in streaks at $1 / 3$ order positions in LEED. Depending on $\mathrm{Na}$ concentration, average $\mathrm{Na}$ chain distances between 4 and 8 have been found, which form commensurate structures at chain separations of $4 a, 6 a$, and $8 a$. The relatively high energy of activation for formation of these structures can be rationalized by the necessary replacement of Ge-dimer rows by $\mathrm{Na}$ chains. The effective lateral interactions between the Na chains, which are of remarkably long range, must be mediated by surface relaxations in the topmost Ge layers coupled with corresponding rearrangements of the electron density. Although the low-coverage structures obtained after adsorption at room temperature turn out to be metastable, and although the structures found are strongly anisotropic, the structural rearrangement necessary to obtain

*Electronic address: pfnuer@ fkp.uni-hannover.de

${ }^{1}$ A. H. Somer, Photoemissive Materials (Wiley, New York, 1968).

${ }^{2}$ J.E. Ortega, E.M. Oellig, J. Ferron, and R. Miranda, Phys. Rev. B 36, 6213 (1987).

${ }^{3}$ M. Tikhov, G. Rangelov, and L. Surnev, Surf. Sci. 231, 280 (1990).

${ }^{4}$ G. Faraci and A.R. Pennisi, Surf. Sci. 409, 46 (1998).

${ }^{5}$ S.Y. Davydov, Appl. Surf. Sci. 140, 58 (1999).

${ }^{6}$ A.A. Saranin, A.V. Zotov, S.V. Ryzhkov, D.A. Tsukanov, V.G. Lifshits, J.T. Ryu, O. Kubo, H. Tani, T. Harada, M. Katayama, and K. Oura, Phys. Rev. B 58, 7059 (1998).

${ }^{7}$ A.A. Saranin, A.V. Zotov, V.G. Lifshits, M. Katayama, and K. Oura, Surf. Sci. 426, 298 (1999).

${ }^{8}$ A.A. Saranin, V.G. Lifshitz, K.V. Ignatovich, H. Bethge, R. Kayser, H. Goldbach, A. Klust, J. Wollschläger, and M. Henzler, Surf. Sci. 448, 87 (2000).

${ }^{9}$ A.A. Saranin, A.V. Zotov, S.V. Ryzhkov, D.A. Tsukanov, V.G. Lifshits, J.T. Ryu, O. Kubo, H. Tani, T. Harada, M. Katayama, and K. Oura, Phys. Rev. B 58, 4972 (1998).

${ }^{10}$ S.V. Ryjkov, T. Nagao, V.G. Lifshits, and S. Hasegawa, Surf. Sci. 493, 619 (2001).

${ }^{11}$ O. Gallus, T. Pillo, M. Hengsberger, P. Segovia, and Y. Baer, Eur. the more stable $n \times 3$ structures does not fit to the classical scenario of a Peierls instability ${ }^{20}$ but details about the Nainduced electronic changes in this system have not yet been investigated.

The minority species of $\mathrm{Na}$, which is adsorbed on doubledimer rows of Ge, may be identical to the species obtained after room temperature adsorption, but no systematic investigations have been made to further identify this species.

In contrast to the $\mathrm{Na}(2 \times 3)$ structure, these Na-induced chain structures have a marginal effect on the step morphology. The reason may be the mixture of Ge dimer and $\mathrm{Na}$ rows, with a dominant contribution from the Ge-dimer rows, i.e., mainly an effect of concentration, but also an influence by varying local adsorption geometries, as suggested by the geometrical models of the $(6 \times 3)$ (see above) and the $(2$ $\times 3$ ) structure, ${ }^{19}$ respectively.

These Na-induced modulations of the $\mathrm{Ge}(100)$ surface have been shown to be able to also modulate epitaxial layers adsorbed on top of this surface. ${ }^{15}$ This in an example how adsorbate induced self-organized restructuring of a surface can be used as template to impose well-ordered modulations even onto an insulating film.

\section{ACKNOWLEDGMENTS}

It was a pleasure to acknowledge the support for this collaboration by K.-H. Meiwes-Broer and stimulating discussions with him. This work was in part supported by the Deutsche Forschungsgemeinschaft.

Phys. J. B 20, 313 (2001).

${ }^{12}$ C. Kumpf, O. Bunk, J.H. Zeysing, Y. Su, M. Nielsen, R.-L. Johnson, R. Feidenhansl, and K. Bechgaard, Phys. Rev. Lett. 85, 4916 (2000).

${ }^{13}$ H.W. Yeom, S. Takeda, E. Rotenberg, I. Matsuda, K. Horikoshi, J. Schaefer, C.M. Lee, S.D. Kevan, T. Ohta, T. Nagao, and S. Hasegawa, Phys. Rev. Lett. 82, 4898 (1999).

${ }^{14}$ S.S. Lee, J.R. Ahn, N.D. Kim, C.G. Hwang, J.W. Chung, H.W. Yeom, S.V. Ryikov, and S. Hasegawa, Phys. Rev. Lett. 88, 196401 (2002).

${ }^{15}$ W. Ernst, M. Eichmann, H. Pfnür, K.-L. Jonas, V. von Oeynhausen, and K.H. Meiwes-Broer, Appl. Phys. Lett. 80, 2595 (2002).

${ }^{16}$ L. Spiess, E. Wimmer, and P. Soukiassian, Appl. Surf. Sci. 92, 501 (1996).

${ }^{17}$ B. Naydenov and L. Surnev, Surf. Sci. 370, 155 (1997).

${ }^{18}$ H.L. Meyerheim, R. Sawitzki, and W. Moritz, Phys. Rev. B 52, 16830 (1995).

${ }^{19}$ W. Ernst, C. Tegenkamp, H. Pfnür, K.-L. Jonas, V. Von Oeynhausen, and K.-H. Meiwes-Broer, Surf. Sci. 540, 303 (2003).

${ }^{20}$ G. Grüner, Density Waves in Solids (Addison-Wesley, Redwood City, 1994). 